Artículos originales

\title{
Estado visual en niños con parálisis cerebral en el Centro de Rehabilitación e Inclusión Infantil Teletón Aguascalientes
}

\section{Visual status in children with cerebral palsy at Rehabilitation and Inclusion Child Center Teleton, Aguascalientes}

Cornejo-Rubalcava, María Fernanda; Zamilpa-Velázquez, Flor de Rocío; Muñoz-Guerrero, David; Casillas-Casillas, Elizabeth; Ramírez-González, Sergio

\begin{abstract}
María Fernanda Cornejo-Rubalcava
Universidad Autónoma de Aguascalientes, México

Flor de Rocío Zamilpa-Velázquez

Universidad Autónoma de Aguascalientes, México David Muñoz-Guerrero

Universidad Autónoma de Aguascalientes, México

Elizabeth Casillas-Casillas

elizabeth.casillas@edu.uaa.mx

Universidad Autónoma de Aguascalientes, México Sergio Ramírez-González

Universidad Autónoma de Aguascalientes, México
\end{abstract}

\author{
Lux Médica \\ Universidad Autónoma de Aguascalientes, México \\ ISSN: 2007-1655 \\ Periodicidad: Cuatrimestral \\ vol. 17, núm. 49, 2022 \\ luxmedica.editorial@gmail.com \\ Recepción: 20 Julio 2021 \\ Aprobación: 20 Octubre 2021 \\ URL: \\ https://revistas.uaa.mx/index.php/luxmedica/article/view/3274 \\ DOI: \\ https://doi.org/10.33064/491m20223274
}

Resumen: Introducción: Estrabismo, ambliopía y nistagmo son hallazgos visuales frecuentes en niños con parálisis cerebral. Se han reportado insuficiencia de acomodación, disfunción oculomotora y el estado refractivo más frecuente es la hipermetropía. Los niños con discapacidades motoras graves tienen un mayor riesgo de presentar déficits visuales. Objetivo: Evaluar el estado visual en niños con diagnóstico de parálisis cerebral (PC). Métodos: Estudio observacional y descriptivo en 112 pacientes del Centro de Rehabilitación e Inclusión Infantil Teletón (CRIT Aguascalientes). Resultados: El 69\% de sexo masculino y el 31\% femenino. El 55\% presentó hipermetropía; el 10\%, emetropía; el $12 \%$, astigmatismo miópico compuesto; el 17\%, astigmatismo hipermetrópico compuesto; el 4\%, miopía; el 2\%, astigmatismo miópico simple. El $44 \%$ presentó estrabismo, de los cuales el $21 \%$ padecía endotropías; el 23\%, exotropías, y el 7\%, nistagmo. El 30\% reportó fusión y estereopsis; al $42 \%$ se le sugirió terapia visual; al $20 \%$, corrección óptica y terapia; al $4 \%$ terapia y oclusión, y al $10 \%$ solo corrección óptica. El 19\% no requirió ningún tratamiento optométrico. Conclusión: La PC se presentó en mayor porcentaje en el sexo masculino. El 44\% presenta buena agudeza visual; en el $55 \%$ de los casos se encontró hipermetropía; en el $44 \%$, estrabismo, la exotropía más frecuente que endotropía; nistagmo en el $4 \%$ de los casos; en el 45\%, disfunción oculomotora; en el 30\%, fusión y estereopsis. En el $43 \%$ se indicó terapia visual en casa; en el $20 \%$, corrección óptica y oclusión. Posterior a la intervención optométrica se observó un mejor desempeño visual en el 100\% de los casos analizados.

Palabras clave: Parálisis cerebral, visión binocular, estado visual en niños.

Abstract: Background: Strabismus, amblyopia and nystagmus are common visual findings in children with cerebral palsy (CP), accommodative insufficiency, oculomotor dysfunction and the most frequently refractive error reported is hyperopia. Children with severe motor disabilities are said to be at increased risk of visual deficits. Objective: Assessing visual status in children 


\begin{abstract}
diagnosed with cerebral palsy (CP) Methods: An observational, descriptive study was conducted on 112 patients at Rehabilitation and Inclusion Center, Teletón, (CRIT Aguascalientes). Results: $69 \%$ were male and $31 \%$ female. 55\% had hyperopia, $10 \%$ emmetopia, $12 \%$ myopic astigmatism, 17\% hypermetropic astigmatism, $4 \%$ myopia, $2 \%$ simple myopic astigmatism. $44 \%$ had strabismus, of which $21 \%$ were esotropia, $23 \%$ exotropia and $7 \%$ nystagmus. $30 \%$ reported fusion and stereopsis. $42 \%$ were suggested visual therapy, 20\%optical correction and visual therapy, $4 \%$ visual therapy and occlusion, $10 \%$ only optical correction and $19 \%$ did not require any optometric treatment. Conclusion: CP was presented in a higher percentage in the male, $44 \%$ have good visual acuity, $55 \%$ of cases hyperopia, $44 \%$ strabismus, exotropia slightly higher than esotropia, nystagmus in $4 \%$ of cases, $45 \%$ oculomotor dysfunction, $30 \%$ fusion and stereopsis, $43 \%$ was indicated home visual therapy $20 \%$ optical correction and occlusion. After the optometric intervention, a better visual performance was observed in $100 \%$ of the cases analyzed. Introducción
\end{abstract}

\title{
Introducción
}

La parálisis cerebral (PC) involucra un grupo de alteraciones del desarrollo, específicamente las relacionadas con la actividad motora y con la postura, que tienen como consecuencia la deficiencia en la actividad física ocasionada por una lesión a nivel cerebral no progresiva. Esto puede presentarse desde los primeros meses de vida intrauterina o, bien, durante la infancia. Estas alteraciones motoras con frecuencia están asociadas a manifestaciones en la sensación, percepción, deficiencias cognitivas y problemas de comunicación, así como trastornos en la conducta, ya sea por epilepsia o secundarios a problemas musculoesqueléticos. Es la principal causa de discapacidad infantil, según lo reportado por la Revista Mexicana de Ortopedia Pediátrica. ${ }^{1}$ Clínicamente, se puede clasificar en tres categorías: espástica, discinética y atáxica. Cada una de las categorías afecta a diferentes áreas del cerebro y pueden presentar manifestaciones muy variadas. El mayor porcentaje se clasifica en la categoría espástica, con una frecuencia de entre 75 y $80 \%$; mientras que el resto se divide en los otros dos tipos. ${ }^{1}$ La variedad discinética presenta movimientos extrapiramidales debido a regulaciones anormales en el tono, dificultad en el control de la postura y deficiencia en la coordinación. Los movimientos discinéticos se definen como atetosis; su principal característica es que son involuntarios y muy lentos, parecen imitar la escritura e involucran la participación tanto de músculos agonistas como antagonistas. También se presenta otro tipo de movimiento conocido como corea; un tipo de movimiento irregular, abrupto, involuntario y de gran amplitud, principalmente de la cabeza, o el cuello y extremidades. Cuando se presenta la combinación de ambos se denomina coreoatetósicos. El patrón dominante es el atetósico.

Otro tipo de movimientos son los llamados distonía, son movimientos del tronco y extremidades, rítmicos y lentos lo cual provoca que se adopten posturas anormales o inestabilidad llamada ataxia, estos movimientos pueden estar asociados con nistagmo, dismetría y marcha con gran soporte. La parálisis 
cerebral también se puede clasificar anatómicamente en: hemiplejía, diplejía y cuadriplejía. ${ }^{1}$

La hemiplejía afecta un solo lado del cuerpo y se presenta entre un $20 \%$ y un $30 \%$ de los casos. La frecuencia de la diplejía es de un $60 \%$ a un $70 \%$ de los casos; se refiere a la disminución de la habilidad en las extremidades superiores o con más frecuencia las inferiores. Y la presentación más severa es la cuadriplejía con una frecuencia del $10 \%$ al $15 \%$ y afecta a las cuatro extremidades. ${ }^{2}$

El daño cerebral provocado por esta condición, puede afectar las vías visuales en todo su trayecto hasta la corteza, así como a las áreas de asociación visual. Esto puede dar como resultado una variedad de alteraciones visuales que pueden ser una baja agudeza visual, disminución del campo visual, disfunción oculomotora, deficiencias en la integración sensorial y alteraciones de la percepción visual. ${ }^{3}$ Un daño en el sistema oculomotor puede ocasionar anormalidades en las funciones de fijación, movimientos de seguimiento y movimientos sacádicos, estrabismo y movimientos oculares anormales. ${ }^{3}$ Se presenta también un impedimento visual perceptual, como déficit de la función visual causado por un daño o malformación de las vías visuales retrogeniculadas (que incluyen las radiaciones ópticas, la corteza occipital y las áreas de asociación) en ausencia de cualquier enfermedad mayor. ${ }^{4}$

A nivel mundial, su incidencia aproximada es de 2-3 por cada 1,000 nacidos vivos. La población mundial con parálisis cerebral excede los 17 millones de personas. $^{5}$

En México, según lo reportado por la Revista Médica del Instituto Mexicano del Seguro Social, alrededor del 10\% de los pacientes con discapacidad tienen el diagnóstico de parálisis cerebral. De ellos, más del 60\% de los casos son de tipo espástico. ${ }^{5}$

En el CRIT de la Ciudad de México, de 1,112 historias clínicas de pacientes con daño neurológico revisadas, 773 fueron de pacientes diagnosticados con parálisis cerebral, 653 presentaron factores de riesgo prenatales en el tercer trimestre de embarazo y al momento del parto, y 120 presentaron factores de riesgo posnatal. Predominó el sexo masculino y la edad de los padres y la puntuación APGAR no parecen influir en el diagnóstico. ${ }^{6}$

En el CRIT de Aguascalientes, durante el periodo de 2003 a 2013 se registró una población de 1,002 pacientes -algunos han sido egresados o permanecen activos-, de los cuales 850 fueron diagnosticados con parálisis cerebral. Predominó el tipo espástico en un 76\% (646 pacientes), el tipo discinético en el $18.4 \%$ (157 pacientes), el hipotónico en un 4.3\% (37 pacientes) y el atáxico en un $1.1 \%$ (10 pacientes). ${ }^{1}$

La clínica de atención del neurodesarrollo en Aguascalientes, México, reportó una incidencia de trastornos motores, un $70 \%$ presenta disminución de la agudeza visual, el 50\% estrabismo, y algunos presentan casos de nistagmo, ametropías, defectos en campos visuales e incluso ceguera. ${ }^{7}$

Otro estudio realizado en el hospital nacional de neurología y neuropediatría abarcó las áreas motoras, sensoriales y refractivas en 48 pacientes con estrabismo secundario a daño cerebral de 1 a 10 años. Se realizó neurorehabilitación con toxina botulínica como elemento relajante muscular para la recuperación de las funciones visuales. Esto se promueve como una alternativa de menor invasión en 
pacientes con daño cerebral y se sugiere que la mejoría visual puede repercutir en la mejora de otras funciones cerebrales, especialmente la visuopercepción, que a su vez mejora el aprendizaje y la memoria. ${ }^{8}$

Dentro de los hallazgos oculares y visuales, los niños con parálisis cerebral de tipo espástico tienen una alta prevalencia de estrabismo, sobre todo exotropías o desviaciones divergentes, y endotropías o desviaciones convergentes de ángulo grande. En cuanto al estado refractivo, se encuentra con mayor frecuencia la hipermetropía, en la cual, los rayos de luz que inciden en el ojo enfocan un plano posterior a la retina.?

La ambliopía, fue definida por Ciuffreda como el desarrollo anormal de la visión debido a una alteración fisiológica en el córtex visual. Según la Academia Americana de Oftalmología (AAO), consiste en una disminución unilateral, con menor frecuencia bilateral, y suele aparecer desde el nacimiento hasta los primeros 7-8 años de vida. ${ }^{9}$ El nistagmo, o movimiento involuntario de los ojos, es común, por lo que resulta indispensable una evaluación optométrica detallada. Hay evidencia reciente que afirma que niños con discapacidades motoras más graves tienen un mayor riesgo de padecer déficits acomodativos. Se ha demostrado que la parálisis cerebral tiene un impacto negativo en la acomodación ocular. ${ }^{10}$ No obstante, muchos niños con PC tienen trastornos de la percepción visual-espacial a pesar de la agudeza visual esperada. ${ }^{11}$

En México, la PC comenzó a tomar importancia hacia el año 1947. Cuando se incluyó la rehabilitación de pacientes con parálisis cerebral, se observó que presentaban mejoría importante en otras áreas como en el lenguaje y habilidades motoras espontáneas que les permitían deambular. Estos cambios fueron percibidos primeramente por los padres y, posteriormente, se inició el manejo multidisciplinario de pacientes con parálisis cerebral en el Hospital Infantil de México. Se hizo énfasis en el diagnóstico y estimulación temprana, tomando en cuenta las posibles causas prenatales, natales o postnatales, difundiéndose a otros centros hospitalarios nacionales y algunos de Latinoamérica. ${ }^{12}$

La rehabilitación de un niño con parálisis cerebral pretende educar e integrarlo al medio familiar y social. Se trabaja en equipo interdisciplinario, conjuntamente con sus padres, utilizando los recursos remanentes para que desarrolle todas sus potencialidades físicas, mentales, emocionales, sociales y económicas. ${ }^{12}$ Por lo anterior, se planteó como objetivo evaluar el estado visual en niños con diagnóstico de PC que asisten al Centro de Rehabilitación e Inclusión Infantil Teletón de la ciudad de Aguascalientes.

\section{MÉTODOS}

Durante el periodo de agosto a diciembre de 2018, se realizó un estudio observacional descriptivo a una muestra de 112 casos del Centro de Rehabilitación e Inclusión Infantil Teletón Aguascalientes. De un total de 305 pacientes, se descartaron 193 ya que no cumplieron con los criterios de inclusión.

Se incluyeron pacientes con diagnóstico de parálisis cerebral de cualquier tipo, y de sexo y edad indistintos. Previo consentimiento informado, se llevó a cabo una evaluación visual en la que se determinó la agudeza visual lejana y cercana mediante el test de mirada preferencial, New York Lighthouse, LEA 
test y optotipos de Snellen, seleccionándose el procedimiento más adecuado de acuerdo a las características de cada paciente. Se identificó el estado refractivo con la técnica de Mohindra, el alineamiento visual a través de la prueba de oclusión o cover test y mediante la prueba de Hirschberg. Los movimientos oculares de seguimiento se evaluaron con la técnica de SCCO (Southern California College of Optometry System). Los aspectos sensoriales fueron examinados con luces de Worth y la prueba de estereopsis de Lang. Se estableció el diagnóstico refractivo, de salud ocular y visión binocular, y se propuso un plan de manejo optométrico por medio de corrección óptica, oclusión y/o terapia visual. Se analizaron los datos mediante estadística descriptiva.

\section{RESULTADOS}

La muestra estuvo conformada por 112 casos, de los cuales el 69\% corresponde al sexo masculino y el $31 \%$ al sexo femenino (figura 1 ).

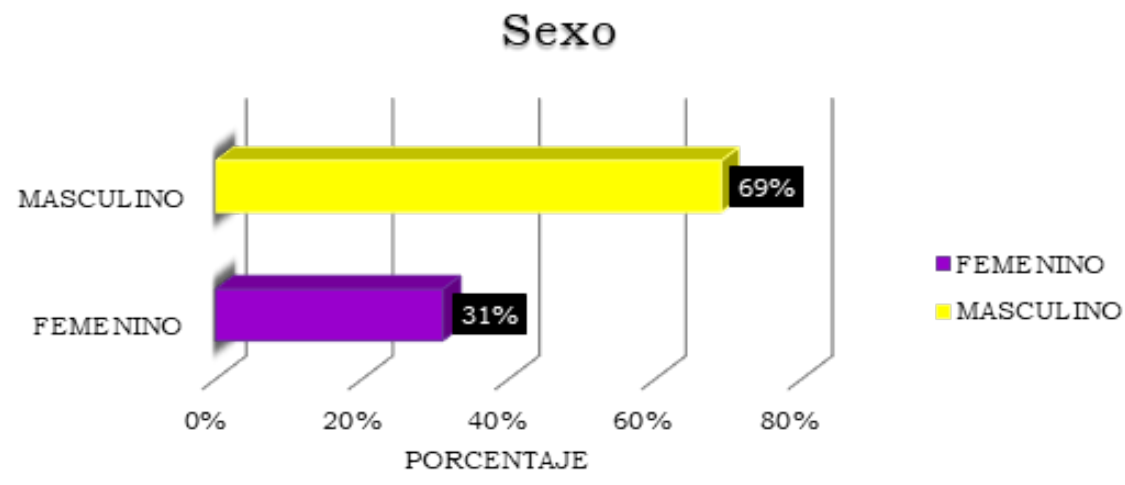

Figura 1. Distribución por sexo

El promedio de edad fue de 4 años (mínima 5 meses y máxima 13 años). En relación al diagnóstico general, el $69 \%$ padecía parálisis cerebral y el $31 \%$ presentó además de PC otro diagnóstico como Síndrome de Moebius o Hidrocefalia, entre otros. Los resultados se presentan en la figura 2.

\section{Diagnôstico General}

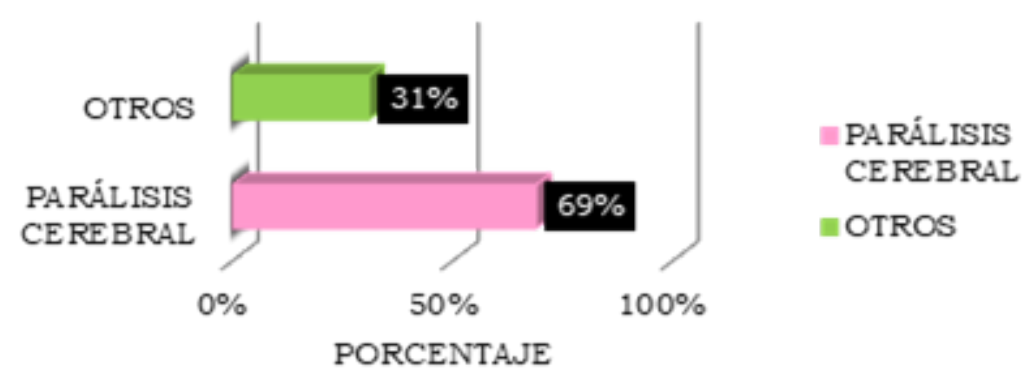

Figura 2. Distribución por diagnóstico general

Con respecto al nivel de agudeza visual, la figura 3 muestra que el $44 \%$ de los casos se registró entre $20 / 20$ y $20 / 40$, el $9 \%$ entre $20 / 50$ y $20 / 80$, el $9 \%$ entre 
20/100 y 20/200 y en el 38\% de los casos no fue posible determinar la visión de manera cuantitativa. Sin embargo, sí se observó respuesta a estímulo luminoso, localización y seguimiento de objetos.

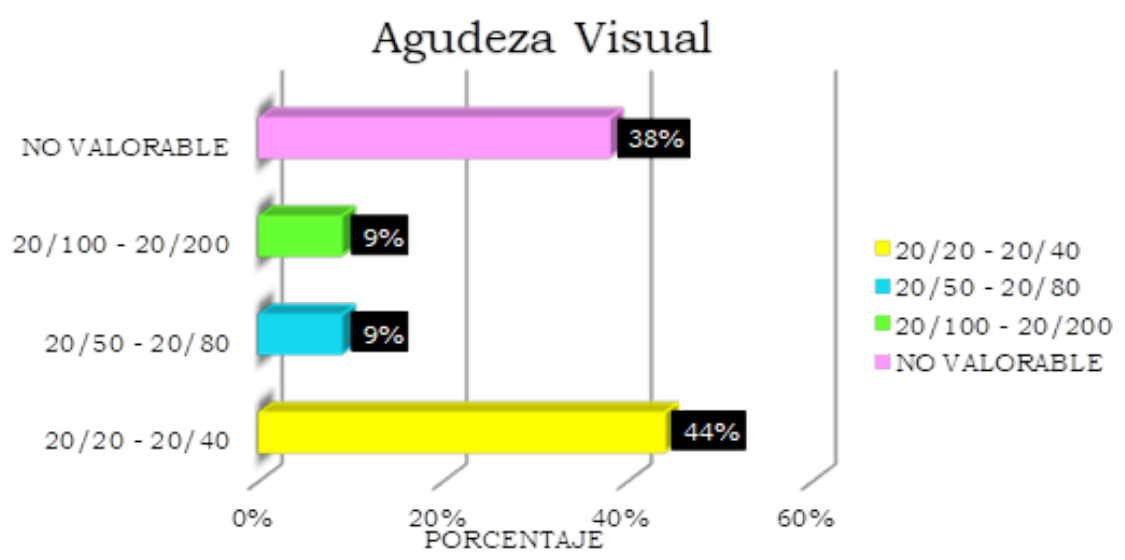

Figura 3. Distribución por nivel de agudeza visual

La figura 4 presenta el estado refractivo encontrado en la muestra el 55\% presentó hipermetropía; el 10\%, emétropes; el 30\%, astigmatismo en sus diversas presentaciones, y el 5\%, miopía.

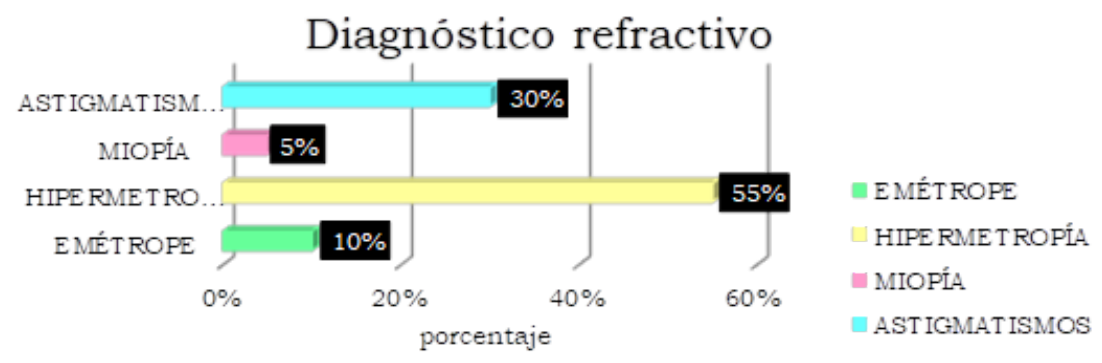

Figura 4. Distribución por diagnóstico refractivo

Con respecto a la posición ocular o alineamiento visual, la figura 5 muestra la distribución de la muestra: el 49\% presentó ortoforia; el 44\%, estrabismo; el 23\%, exotropía; el 32\%, endotropía; el 3\%, nistagmo, y el 4\%, nistagmo combinado con otra condición. 


\section{Alineamiento visual}

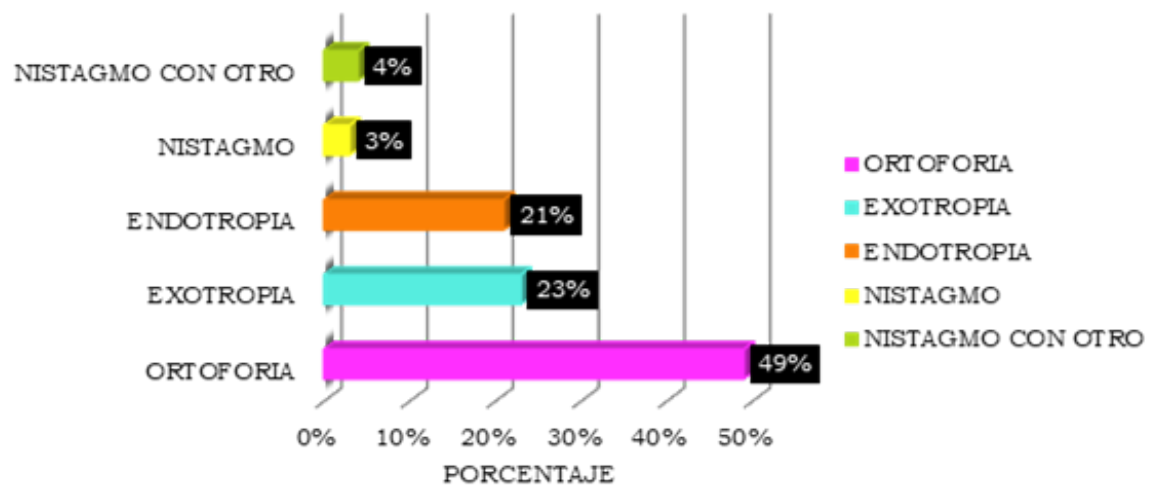

Figura 5. Alineamiento visual o posición ocular

En relación a la motilidad ocular, se evaluaron los movimientos oculares de seguimiento: el 55\% de los casos presentó una adecuada motilidad ocular; el 32\%, disfunción oculomotora, y en el $13 \%$ no fue posible evaluar debido a una pobre fijación en el estímulo utilizado y poca cooperación del paciente. Con respecto al aspecto sensorial, se determinó la presencia de fusión y estereopsis: el 30\% reportó fusión y estereopsis presente, el 16\% ausente y en el $54 \%$ de los casos no se obtuvo la cooperación del paciente para evaluar el aspecto sensorial.

Finalmente, se analizó el tipo de manejo optométrico proporcionado. En la figura 6 se presenta la distribución por tipo de tratamiento: en el $42 \%$ de los pacientes se indicó terapia visual, básicamente se sugirió realizar movimientos de seguimiento ocular y ejercitar la convergencia. Al 20\% se le prescribió la corrección óptica $(\mathrm{RX})$ y se indicaron ejercicios de seguimiento ocular en casa. Del resto, al $4 \%$ se le indicó terapia visual y oclusión; al 10\% solo corrección óptica; al 2\%, oclusión, y al 3\%, oclusión y corrección óptica, mientras que el $19 \%$ no requiere ningún tratamiento optométrico. Posterior a la intervención optométrica, se observó, en el $100 \%$ de los casos, una mejoría en la agudeza visual, en el control oculomotor y, por consecuencia, en el alineamiento visual, presentando una disminución de la frecuencia de la desviación.

\section{Tipo de tratamiento optométrico}

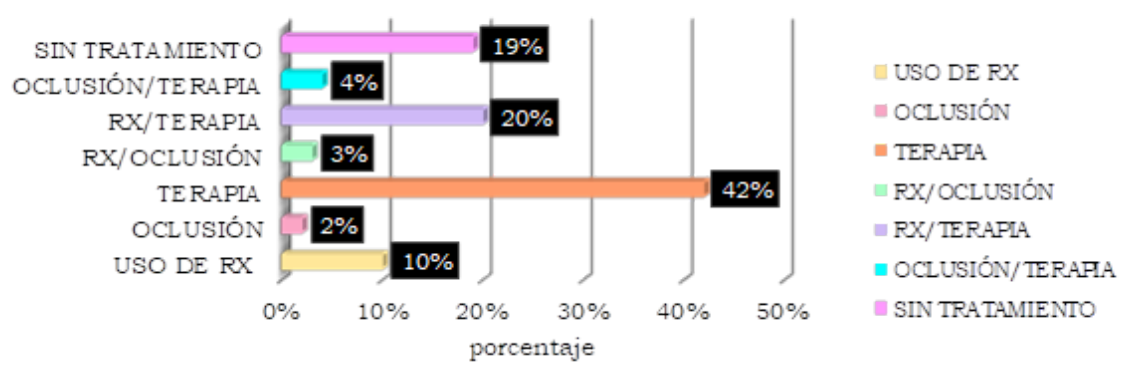

Figura 6. Distribución por tipo de tratamiento optométrico 


\section{DISCUSIÓN}

La muestra estudiada fue de 112 casos, entre los cuales predominó el sexo masculino en un $69 \%$. En el $44 \%$ se determinó una buena agudeza visual y, debido a la condición general, no fue posible cuantificar la agudeza en el $38 \%$ de los casos.

En relación a la posición ocular, el $44 \%$ de la muestra presentó estrabismo, en el $21 \%$ fueron endotropías y en el $23 \%$ exotropías. Estos datos difieren a los encontrados por Lindberg, quién estudió una muestra de 48 niños con parálisis cerebral y afirmó que las endodesviaciones tuvieron una prevalencia del 65\% mientras que las exotropías solo del $35 \% .{ }^{13}$ Esto puede ser debido a la diferencia en el tamaño de la muestra.

Referente al nistagmo, el mencionado estudio lo encontró solo en $8 \%$ del total de casos, similar a lo encontrado en este estudio. Por otra parte, una investigación realizada por Reshma Raj realizada a 20 pacientes con parálisis cerebral y sus tipos, menciona que, respecto a la posición ocular, se encontró en su mayoría pacientes con ortoforia, por lo cual concuerda con los resultados obtenidos de los pacientes en el CRIT. ${ }^{14}$

Con respecto al estado refractivo, la hipermetropía fue la más frecuente, con un $55 \%$ de los casos, lo cual coincide con el estudio citado anteriormente que mostró que el $54 \%$ de los individuos tuvieron un estado refractivo significativo, siendo la hipermetropía mayor a dos dioptrías la más frecuente. ${ }^{1}$

Mientras que otro estudio realizado por Benítez-Nava y colaboradores nos menciona que en la exotropía es más prevalente de acuerdo a la investigación realizada en 73 pacientes, los resultados arrojan que el $20 \%$ de los individuos presentan exotropía, mientras que el $3 \%$ endotropía, lo cual coincide con lo que se concluye en esta investigación. ${ }^{16}$

De la misma manera, otro estudio realizado nos indica que la hipermetropía fue el estado refractivo más común observado en la población de la investigación realizada. ${ }^{17}$

No obstante, se encontraron diferencias con las demás ametropías; ya que la miopía solo se encontró en $4 \%$ del total de casos analizados; incluso el $10 \%$ fue emétrope, el $12 \%$ presentó astigmatismo miópico compuesto, el $17 \%$ tenía astigmatismo hipermetrópico compuesto, y el 2\% padecía astigmatismo miópico simple. $^{17}$

En los casos en los que se pudo evaluar el aspecto sensorial, la fusión y estereopsis estuvo presente en un 30\% de los casos. Los hallazgos precedentes afirman que en la mayoría de casos se encuentra ausente o anormal. 18, 19, 20 Tomando en cuenta a la fusión, un artículo publicado recientemente informa que, de acuerdo a la población valorada, un $55 \%$ de los casos presenta fusión en visión cercana, mientras que la estereopsis se reporta en un $60 \%,{ }^{21}$ lo cual difiere con lo obtenido en este estudio.

En cuanto al plan de manejo proporcionado, en el 47\% de los casos se sugirió la terapia visual en casa, principalmente para mejorar la estabilidad en la fijación y la velocidad y exactitud de los movimientos oculares de seguimiento y sacádicos. Y, posterior a la intervención optométrica, se presentó una mejoría en el desempeño visual en el $100 \%$ de los casos. 


\section{CONCLUSIÓN}

La evaluación del estado visual en pacientes con parálisis cerebral reveló que el $44 \%$ de los casos presentó buena agudeza visual y el 33\% no requiere corrección óptica. El estrabismo se presentó en un $44 \%$ de los pacientes, siendo ligeramente mayor el porcentaje de exotropías con respecto a las endotropías. El nistagmo solo se presentó en un 7\%. El 45\% de los casos presentó disfunción oculomotora y en el 30\% de los casos se determinó la presencia de fusión y estereopsis. El tipo de tratamiento recomendado con mayor frecuencia fue la terapia visual en casa, en el 47\% de los casos, y una combinación de terapia, corrección óptica y oclusión. Posterior al tratamiento, se presentó una mejoría en el desempeño visual en el $100 \%$ de los casos.

\section{Referencias}

1. Calzada Vázquez C, Vidal Ruiz C. Parálisis cerebral infantil: definición y clasificación a través de la historia. Rev Mex Orto Ped.2014;16(1):1-16.

2. M. T, Bartuccio M, Dominick M. Visual Diagnosis and Care of The Patient with Special Needs. Wol Klu/Lipp Will \& Wilk; 2012;1(2): 21-30.

3. Ghasia F, Brunstrom J, Gordon M, Tychsen L. Frequency and Severity of Visual Sensory and Motor Deficits in Children with Cerebral Palsy: Gross Motor Function Classification Scale. Inv Opt \& Vis Sci. 2008;49(2):572-116.

4. Park M, Yoo Y, Chung C, Hwang J. Ocular findings in patients with spastic type cerebral palsy. BMC Opht. 2016;4(1):1-195.

5. Revista Médica IMSS. Pre Med, 2021;59(3).

6. Reyes G, Ibarra DB. Factores de riesgo en niños con parálisis infantil en el centro de rehabilitación infantil Teletón. Rehabilitación. 2006;40(1):14-19.

7. Malagón J. Parálisis cerebral. MEDICINA, 2007;67(1):589-592.

8. Moguel-Ancheita S, Valdés-Barrena A, Padilla-Sánchez FG. Neurorrehabilitación visual de pacientes con daño cerebral usando toxina botulínica. Cir Cir. 2012;80(4):320-326.

9. Wallace DK, Repka MX, Lee KA, Melia M, Christiansen SP, Morse CL. Amblyopia Preferred Practice Pattern. Opht. 2019;125(1):105-42.

10. Fazzi E, Signorini S, La Piana R, Bertone C, Misefari W, \& Galli J. Neuroophtalmological disorders in cerebral palsy: ophthalmological, oculomotor, and visual aspects. Dev Med \& Child Neu. 2012; 1(19):1-736.

11. Mina M. Evolución psicosocial del niño con parálisis cerebral: Una mirada desde la experiencia y la investigación psicológica. Bru Edi. 2010.

12. Del Valle-Morales A, Espinosa-Barragán O, Mendoza AG, Parálisis Cerebral Infantil, su presentación clínica en pacientes atendidos en el CRIT Aguascalientes. Lux Med. 2014;37(1):1-18.

13. Erkkila' H, Lindberg L, Kallio A. Strabismus in children with cerebral dalsv. Acta Oph Sca. 1996; 3(74):636-520.

14. Raj R, Kotian VB. Evaluación ocular de pacientes con parálisis cerebral. Act Sci Oft. 2019;2.8(7):2-5.

15. McClelland J, Parkes J, Hill N, Jackson A, Saunders K. Accommodative Dysfunction in Children with Cerebral Palsy. Inv Op \& Vis Sci. 2006;47(5):1824-30 
16. Benítez-Nava A, Arroyo-Yllanes M, Garrido E. Cirugía y cirujanos. Ac Mex de Cir. 1999;1(2):240-210.

17. Tohen A, Tohen A, Aguilera J. La Parálisis cerebral en México. Púb Méx. 1986.

18. Critten V, Campbell E, Farran E, Messer D. Visual perception, visual-spatial cognition and mathematics. Ass And Pre Chil W Cere Pal. 2018;3(80):181-100.

19. Ego A, Lidzba K, Brovedani P, Belmonti V, Gonzalez S, Boudia B. Visual-perceptual impairment in children with cerebral palsy: asystematic review. Dev Med \& Chi Neu.2015;57(2): 46-51.

20. Kozeis N, Anogeianaki A, Mitova DT, Anogianakis G, Mitov T, Klisarova A. Visual function and visual perception in cerebral palsied children. Opht and Phy Opt. 2007;27(1):44-53.

21. Galli J, Ambrosi C, Micheletti S, Merabet LB, Pinardi C, Gasparotti R, et al. White matter changes associated with cognitive visual dysfunctions in children with cerebral palsy: A diffusion tensor imaging study. Jou of Neu Re. 2018;10(2):24-30. 